\begin{tabular}{|c|l|}
\hline Title & I sol lated attosecond pul se generation by monocycle pumping : the use of a harmonic region with minimum dispersion \\
\hline Author(s) & Fang, Shaobo; Tanigawa, Takashi; I shikawa, Kenichi L.; Karasawa, Naoki; Y amashita, Mikio \\
\hline Citation & $\begin{array}{l}\text { Journal of the Optical Society of A merica B: Optical Phy sics, 28(1), 1-9 } \\
\text { https://doi.org/L0.1364/JOSAB.28.000001 }\end{array}$ \\
\hline Issue Date & 2011-01-01 \\
\hline Doc URL & http://hdl.handle.net/2115/44872 \\
\hline Rights & ○ 2011 Optical Society of A merica \\
\hline Type & article \\
\hline File Information & JOSA B28_1_1-9.pdf \\
\hline
\end{tabular}

Instructions for use 


\title{
Isolated attosecond pulse generation by monocycle pumping: the use of a harmonic region with minimum dispersion
}

\author{
Shaobo Fang, ${ }^{1,4}$ Takashi Tanigawa, ${ }^{1}$ Kenichi L. Ishikawa, ${ }^{2}$ \\ Naoki Karasawa, ${ }^{3}$ and Mikio Yamashita ${ }^{1,5}$ \\ ${ }^{1}$ Department of Applied Physics, Hokkaido University, and Core Research for Evolutional Science and Technology, \\ Japan Science and Technology Agency, Kita-13, Nishi-8, Kita-ku, Sapporo, Hokkaido, 060-8628, Japan \\ ${ }^{2}$ Photon Science Center, Graduate School of Engineering, The University of Tokyo, and Core Research \\ for Evolutional Science and Technology, Japan Science and Technology Agency, \\ 7-3-1 Hongo, Bunkyo-ku, Tokyo, 113-8656, Japan \\ ${ }^{3}$ Chitose Institute of Science and Technology, 758-65 Bibi, Chitose, Hokkaido, 066-8655, Japan \\ ${ }^{4}$ e-mail: fangshao@eng.hokudai.ac.jp \\ ${ }^{5}$ e-mail: mikio@eng.hokudai.ac.jp
}

Received June 18, 2010; revised October 22, 2010; accepted October 24, 2010;

posted October 27, 2010 (Doc. ID 130250); published December 6, 2010

\begin{abstract}
The detailed theoretical investigation into the spectral phase and intrinsic dispersion of high-order harmonics generated by monocycle pumping [a $1.93 \mathrm{fs}\left(T_{0}\right), 580 \mathrm{~nm}\left(\lambda_{0}\right), 1.7-2.0 \times 10^{15} \mathrm{~W} / \mathrm{cm}^{2}\left(I_{0}\right)$, Gaussian-shaped pulse] showed that it is significantly important for further shortening of isolated attosecond pulses to select the spectral region that has the minimum intrinsic group-delay dispersion and higher-order dispersion in the middle region of the plateau, other than that close to the cutoff where the harmonic intensity drops rapidly. We demonstrated that the selection is carried out directly from the harmonic-order-dependent electron-recombination time, which is obtained from the simple semiclassical method and can be measured by experiment. Consequently, we found that monocycle pumping can generate efficiently 68 as isolated pulses without chirp compensation as well as tunable (115 to $150 \mathrm{eV}$ ), sub 70 as pulses from He atoms by tuning the carrier-envelope phase. Particularly, nearly transform-limited 43 as isolated pulses can be produced under the double-intensity $\left(I_{\text {pump }}=2 I_{0}\right)$ and doublewavelength $\left(\lambda_{\text {pump }}=2 \lambda_{0}, T_{\text {pump }}=2 T_{0}\right.$ ) pump. These results were also confirmed by the saddle-point analysis based on the Lewenstein model. (C) 2010 Optical Society of America

OCIS codes: $\quad 190.4160,020.2649,320.0320$.
\end{abstract}

\section{INTRODUCTION}

Generation of isolated attosecond pulses (IAPs) in the extreme ultraviolet (XUV) region through high-order harmonic generation by the interaction of intense ultrashort laser pulses with atoms has highlighted the importance of attosecond science in the new century [1,2]. In 2001, the first generation of the IAP with a 650 as duration in the XUV region was reported using a 2.8 cycle near-infrared (NIR) pulse as an intense ultrashort pump source [3]. After that (2004), by excitation of a 2.0 cycle NIR pulse, they generated a shorter 250 as IAP [4]. On the other hand, a variety of pumping techniques using a longer driving pulse duration, such as polarization gating $[\underline{5}, \underline{6}]$, two-color gating $[\underline{7}, \underline{8}]$, ionization gating $[\underline{9}, \underline{10}]$, and long-wavelength pumping $[\underline{11}, \underline{12}]$ were theoretically proposed and experimentally attempted. Among them, Sansone et al. have reported the generation of a 130 as isolated XUV pulse using a 2.0 cycle NIR driving pulse with a modulated polarization state [6]. Moreover, for the sub 100 as pulse generation, different multicolor gating methods, including the superposition of the weak third-order harmonic field and/or the dc field with a main driving pulse, have been theoretically proposed [13-15]. In addition, the experiment by the combined method of the two-color gating and the polarization gating was implemented [16].
In spite of those efforts, using only a single $3.3 \mathrm{fs}$, sub 1.5 cycle NIR pump pulse, it was reported that a sub 100 as isolated XUV pulse (an 80 as pulse, the shortest pulse to date) was generated [17], which shows brightness in monocycle pumping. However, because of the limitation of the currently available shortest pump pulse duration with chirped-mirror compensation, it is difficult to offer a shorter attosecond pulse by this approach. On the other hand, $2.6 \mathrm{fs}$, 1.3 cycle isolated pulses were generated by compensating for the chirp of the ultrabroadband pulses generated by inducedphase modulation and self-phase modulation using a spatial light modulator [18]. It was also demonstrated experimentally that angularly dispersed noncollinear optical parametric amplification is an attractive way to obtain high-energy over-oneoctave-spanning broadband pulses in the monocycle region [19]. These works have paved the way for monocycle pumping and resulted in shedding light on theoretically and quantitatively monocycle-pumped harmonic phenomena, including the IAP generation.

Up to now, a lot of investigations for the generation of the shorter IAP by few-cycle pumping have been performed by extending the cutoff region to the higher frequency and by broadening the bandwidth while keeping the uniform intensity spectrum [3-17,20-22]. In this paper, we clarify theoretically and quantitatively the spectral phase and intrinsic dispersion of high-order harmonics under monocycle pumping and the 
dependence of the isolated pulse profile on the selected spectral region. Especially, we show the importance of the region with the minimum group-delay dispersion (GDD) and high-order dispersion for the shortest attosecond pulse generation, which is located not at the region close to the cutoff but at the middle region of the plateau where the harmonic intensity is much higher. This is also significantly important from the point of view of the present technical situation that the nonlinear chirp compensation over the entire spectral range of the XUV supercontinuum produced by monocycle pumping is very difficult.

\section{THEORETICAL MODELS FOR NUMERICAL CALCULATION}

Recently, interesting theoretical work on the relation between the microscopic and macroscopic nonlinear response for the harmonic generation has been reported by Li et al. [22]. They have clarified that the harmonics emitted by single atoms can be maintained after propagation through the gas jet when the phase matching of the short electron trajectory is satisfied (see Section 4). This phase-matching technique has usually been employed for the attosecond pulse generation and the measurement of the harmonic dispersion [3, $\underline{4}, \underline{6}, \underline{8}, \underline{17}, \underline{21}-27]$. Therefore, in this paper we studied the nonlinear polarization response of the medium using the following single-atom models: a direct calculation of the time-dependent dipole moment $x(t)$ [Eq. (8) in [28]: see the next paragraph] derived from the widely used Lewenstein model under the strong field approximation (Section 3), a calculation from a quantum orbit model with the use of the saddle-point analysis (SPA) based on the Lewenstein model [29] (Sections 3 and 4 ), and an $a b$ initio calculation of the time-dependent Schrödinger equation (TDSE) [30] for confirmation. The SPA extracts information about each electron trajectory separately, and the TDSE approach provides a full description of the system.

By Fourier transform of the following atomic dipole moment $x(t)$ obtained from the Lewenstein model under the assumption of no ground-state depletion $\left\{|a(t)|^{2}=1\right.$ : Eq. (3) in [28]: $\left.|\Psi(t)\rangle=e^{i I_{p} t}\left(a(t)|0\rangle+\int \mathrm{d}^{3} \mathbf{v} b(\mathbf{v}, t)|\mathbf{v}\rangle\right)\right\}$, we calculated the high-order harmonic intensity spectrum $I(\omega)$ and the spectral phase $\phi(\omega)$ :

$$
\begin{aligned}
x(t)= & i \int_{0}^{t} \mathrm{~d} t^{\prime} \iint_{-\infty}^{\infty} \mathrm{d}^{3} \mathbf{p} d_{x}^{*}(\mathbf{p}-\mathbf{A}(t)) \times E\left(t^{\prime}\right) d_{x}\left(\mathbf{p}-\mathbf{A}\left(t^{\prime}\right)\right) \\
& \times \exp \left[-i \int_{t^{\prime}}^{t} \mathrm{~d} t^{\prime \prime}\left(\frac{\left[\mathbf{p}-\mathbf{A}\left(t^{\prime \prime}\right)\right]^{2}}{2}+I_{p}\right)\right]+c . c .
\end{aligned}
$$

Here we assumed a linearly polarized Gaussian electric field $E(t)$ as the monocycle pumping laser pulse

$$
E(t)=E_{0} \exp \left(-\frac{(2 \ln 2) t^{2}}{T_{1}^{2}}\right) \cos \left[\omega_{0} t+\varphi_{0}\right],
$$

and $\mathbf{p}=\mathbf{v}+\mathbf{A}(t), \mathbf{A}(t)=(A(t), 0,0)$, and $E(t)=-\partial A(t) / \partial t$. The atomic dipole matrix element $d_{x}(\mathbf{p})$ was obtained using the Gaussian model as the $s$-wave function of the ground state and a truncated harmonic oscillator as the atomic potential [28]. Here $\omega_{0}$ is the central angular frequency (corresponding to the central wavelength of $\lambda_{0}=580 \mathrm{~nm}$ ) of the driving pulse and $T_{0}=1.93 \mathrm{fs}$ is the full width at half-maximum (FWHM) of the monocycle pump pulse. The central wavelength was decided based on our experimental result (the 2.8 fs pulse) [31]. The helium atom was chosen as the nonlinear medium of which the ground-state depletion is negligible (the ionization potential $I_{p}=24.59 \mathrm{eV}$ ). To investigate the exact waveform effect of the pump electric field by changing the carrier-envelope phase (CEP) $\left(\varphi_{0}\right)$ from $0.0 \pi$ to $-0.9 \pi$, we kept the maximum value of the electric field constant. Therefore, we slightly changed the peak intensity defined by the envelope from $1.7 \times 10^{15} \mathrm{~W} / \mathrm{cm}^{2}$ for $\varphi_{0}=0.0 \pi$ to $2.0 \times 10^{15} \mathrm{~W} / \mathrm{cm}^{2}$ for $\varphi_{0}=-0.5 \pi$ (and from $2.0 \times 10^{15} \mathrm{~W} / \mathrm{cm}^{2}$ for $\varphi_{0}=-0.5 \pi$ to $1.7 \times 10^{15} \mathrm{~W} / \mathrm{cm}^{2}$ for $\left.\varphi_{0}=-\pi\right)$.

The intensity spectrum $I_{i-j}(\omega)$ and the spectral phase $\phi_{i-j}(\omega)$ (see Section 3 ) by the individual short and long electron trajectories ( $i=\mathrm{S}$ and $\mathrm{L}$ ) of different pairs of quantum orbits ( $i=\mathrm{A}, \mathrm{B}, \mathrm{C}$, and D) were calculated separately by the SPA using the following equation $x_{s}(\omega)$ \{Eq. (3) in [29]\} with the assumption that $|a(t)|^{2}=1$ :

$$
\begin{aligned}
x_{s}(\omega)= & \frac{2 \pi i}{\left.\left[\operatorname{det} S^{\prime \prime}\left(t, t^{\prime}\right)\right]^{1 / 2}\right|_{t_{s}(\omega), t_{s}^{\prime}(\omega)}} \\
& \times\left[\pi /\left(\varepsilon+\frac{i}{2}\left(t_{s}(\omega)-t_{s}^{\prime}(\omega)\right)\right)\right]^{3 / 2} \\
& \times d_{x}^{*}\left[\mathbf{p}_{s}(\omega)-\mathbf{A}\left(t_{s}(\omega)\right)\right] \times E\left(t_{s}^{\prime}(\omega)\right) \\
& \times d_{x}\left[\mathbf{p}_{s}(\omega)-\mathbf{A}\left(t_{s}^{\prime}(\omega)\right)\right] \\
& \times \exp \left[-i\left(S\left(t_{s}(\omega), t_{s}^{\prime}(\omega)\right)+\omega t_{s}(\omega)\right)\right] .
\end{aligned}
$$

The calculation of the three-dimensional TDSE in the length gauge within the single-active-electron approximation was performed by the numerical integration using the alternating direction implicit (Peaceman-Rachford) method [30].

Furthermore, we proposed the recombination time method (see Section 4) using the simple semiclassical three-step model [32] for the evaluation of the harmonic dispersion effect as follows: we calculated the recollision energy $E_{K}\left(t, t^{\prime}\right)$ of the short and long electron trajectories of different pairs of orbits and the photon energy $N\left(t^{\prime}\right)$ or $N(t)$ [hereafter the harmonic order $N\left(t^{\prime}\right)$ or $N(t)$ denotes the photon energy, $N=1$ corresponds to $2.14 \mathrm{eV}$ ] of the emitted harmonics upon recollision as a function of the ionization time $t^{\prime}$ or a function of the recombination time $t$ (see Sections 3 and 4 ). The emitted photon energy is described by $N\left(t^{\prime}\right)=1.3 I_{P}+E_{K}\left(t=g\left(t^{\prime}\right), t^{\prime}\right)$ or $N(t)=1.3 I_{P}+E_{K}\left(t, t^{\prime}=h(t)\right)$ [28]. Here we added the correction factor 1.3 in front of the ionization potential energy $I_{p}$, resulting from the effects of quantum tunnelling and quantum diffusion [28]. The electron recollision energy is given by $E_{K}\left(t, t^{\prime}\right)=\overline{0.5}\left(A(t)-A\left(t^{\prime}\right)\right)^{2}$ using the initial conditions of the electron velocity $\mathbf{v}\left(t^{\prime}\right)=0$ and the electron position $\mathbf{x}(t)=$ $\mathbf{x}\left(t^{\prime}\right)=0$ and the vector potential $A(t)=-\int_{-\infty}^{t} E\left(t^{\prime \prime}\right) \mathrm{d} t^{\prime \prime}$. The relation $\left[t=g\left(t^{\prime}\right), t^{\prime}=h(t)\right]$ between $t$ and $t^{\prime}$ is determined from the equation $B\left(t^{\prime}\right)-t^{\prime} A\left(t^{\prime}\right)+t A\left(t^{\prime}\right)=B(t)$, which is obtained from the initial condition $\mathbf{v}\left(t^{\prime}\right)=0$. Here $B(t)=$ $\int_{-\infty}^{t} A\left(t^{\prime \prime \prime}\right) \mathrm{d} t^{\prime \prime \prime}$. The relation between the recombination time $t$ and the emitted photon energy $N(t)$ is very important for providing information not only on the time-frequency relation of an emitted harmonic photon but also on the intrinsic group delay in the harmonic emission processes [23]. This is because the inverse function $t(N)$ of the recombinationtime-dependent harmonic energy $N(t)$ corresponds to the 
frequency-dependent group delay $t_{\mathrm{DG}}(\omega)$ in the field of femtosecond optics $[31,33,34]$. Accordingly, the first derivative of the harmonic-order-dependent recombination time $t(N)$ with respect to the harmonic frequency $N, \phi^{\prime \prime}(N)=d t(N) / d N$, describes the intrinsic GDD, which is the most important parameter for the attosecond pulse broadening.

\section{CHARACTERISTICS OF HARMONICS GENERATED BY MONOCYCLE PUMPING}

First of all, we investigated the CEP dependence of the harmonic spectral intensity $I(\omega)$ (black curves) and the spectral phase $\phi(\omega)$ (thin blue curves, red curves for enlarged ones, and the red dashed arrows denote the eye-guided shifts in Fig. 1; see Section 2). We find from Fig. 1 that the spectra $I(\omega)$ (harmonic order of $N=\sim 15$ to $\sim 105$ ) have definitely two or three branches in the plateau to cutoff region, which corresponds to the previously reported result [20]. That is, in the CEP range from $0.0 \pi$ (cosine) to $-0.5 \pi$ (sine), a low-

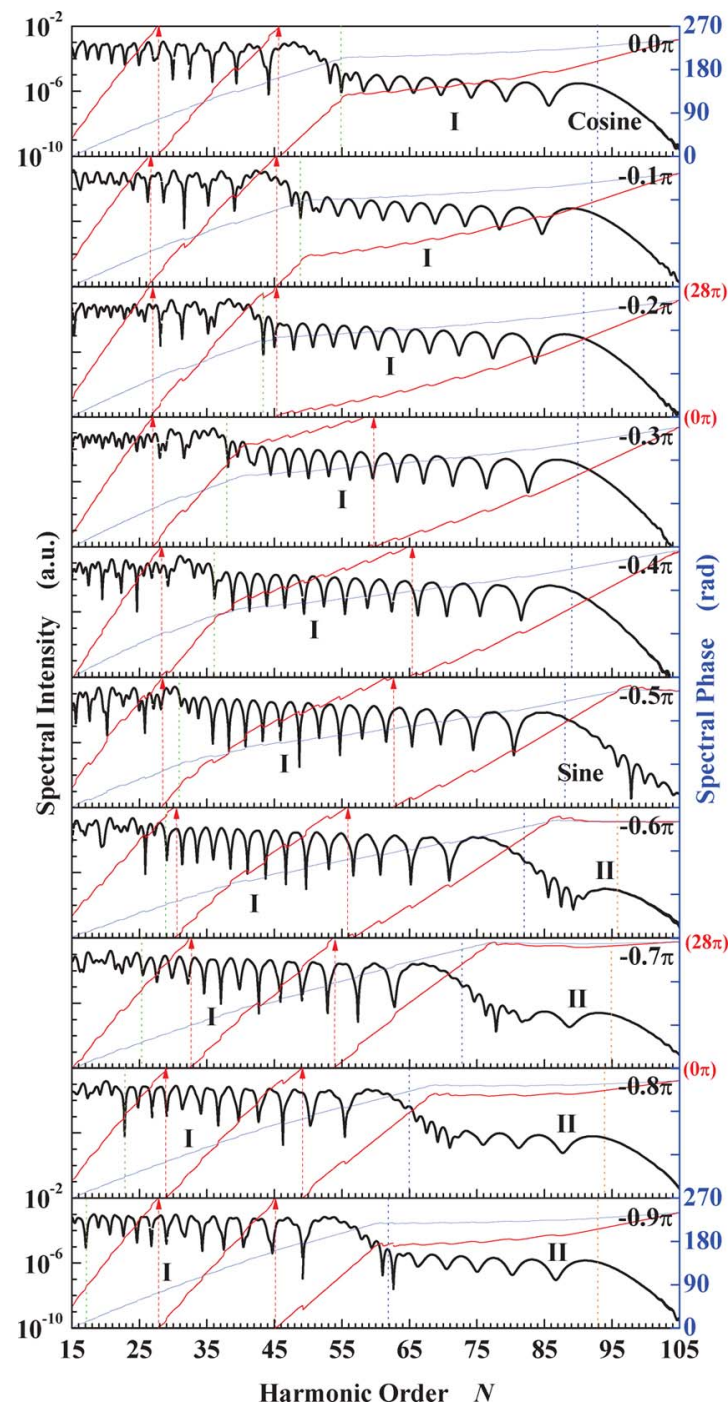

Fig. 1. (Color online) CEP dependence of the spectral intensity (thick black curves) and the spectral phase (thin blue curves and thick red curves for enlarged ones) by monocycle pumping. Here thin dashed red arrows denote the eye-guided shifts employed for the enlargement; the labels (I and II) for the upper branch I and II, respectively; green dotted curves denote the $N_{b}^{I}$, blue dotted curves denote the $N_{\text {peak }}^{I}$, and orange dotted curves denote the $N_{\text {peak }}^{I I}$. intensity upper branch I and a high-intensity lower branch appear, and in the CEP range from $-0.6 \pi$ to $-0.9 \pi$ a new lowerintensity upper branch II appears in the highest harmonic order region in addition to the upper branch I and the lower branch [20]. Furthermore, in monocycle pumping, the regular and slow modulation occurs in the upper-branch spectra, which is different from the spectra with the complicated, branchless structure except for the cutoff region in the few-cycle pumping case $[1,3,4,21,35]$. The boundary $N_{b}^{I}$ between the upper branch I and the lower branch varies from the harmonic order of $N_{b}^{I}=55$ to 32 , and the harmonic order $N_{\text {peak }}^{I}$ at the peak in the cutoff region also slightly shifts from $N_{\text {peak }}^{I}=93$ to 88 , with the CEP varying from $0.0 \pi$ to $-0.5 \pi$. The further CEP change to $-0.9 \pi$ shifts the boundary to $N_{b}^{I}=17$, the peak to $N_{\text {peak }}^{I}=62$, and the new peak of the upper branch II from $N_{\text {peak }}^{I I}=96$ to 94 . The boundaries $N_{b}^{I}$ (or $N_{b}^{I I}$ ) and $N_{\text {peak }}^{I}\left(\right.$ or $\left.N_{\text {peak }}^{I I}\right)$ can be easily estimated by the electron kinetic energy curves as functions of emission and recombination times (see the later description in 3 ).

Next, let us consider the spectral phase behaviors. The boundary $N_{b}^{I I}$ between the upper branches I and II and the boundary $N_{b}^{I}$ between the upper branch I and the lower branch are also observed. The more interesting result is that, in both upper branches, those spectral phases show the piecewise increase dependence (see thin red curve in Fig. 3 for the more enlarged one, $\phi_{S, L}(\omega)=\phi(\omega)$ at CEP $\left.=-0.5 \pi\right)$. The spectral phase envelope increases monotonously but with a series of regular steps at each local minimum of the intensity spectrum. The step interval increases with the increase of the harmonic order (see the later description about Fig. 3 in 3 ). It seems that such spectral phase modulation is a general phenomenon in the coherent light wave interaction based on the multiorder nonlinear optical processes. For example, it was reported that, in the multiple-coherent anti-Stokes Raman scattering from the dielectric crystal, the steplike spectral phase modulation is experimentally observed at each $N$ th order when two different waves are generated by neighbororder nonlinear polarizations $\mathbf{P}^{(N)}$ and $\mathbf{P}^{(N+1)}$ and overlapped spectrally [36]. This is because each nonlinearly induced $N$ thorder polarization $\mathbf{P}^{(N)}$ has a phase shift.

The above-found significant spectral intensity and phase behavior can be well explained by the emitted photon energy upon recollision $\left[N\left(t^{\prime}\right)\right.$ and $N(t)$; see Section 2] as functions of ionization time $\left(t^{\prime}\right)$ [thick black dotted curves in Fig. 2(a) for $\mathrm{CEP}=-0.5 \pi$ ] and recombination time $(t)$ [thick red dotted curves in Fig. 2(a) for CEP $=-0.5 \pi$ ], and by the intensity spectra $I_{i-j}(\omega)$ [in Fig. 2(b) for CEP $=-0.5 \pi$ ] and the spectral phases $\phi_{i-j}(\omega)$ (thick red solid and dotted curves in Fig. 3 for $\mathrm{CEP}=-0.5 \pi$ ) calculated for each single trajectory $i$ of pairs of quantum orbits $j$ of an electron (see Section 2). Let us take up CEP $=-0.5 \pi$ pumping as an example. Figure 2(a) [ $N\left(t^{\prime}\right)$ black curves and $N(t)$ red curves] shows that monocycle pumping mainly produces only three pairs of quantum orbits $[j=\mathrm{B}, \mathrm{C}$, and $\mathrm{D}$ (or A)], and each of them has a pair of short and long electron trajectories ( $j=\mathrm{S}$ and $\mathrm{L})$. In general, the structure of the harmonic intensity spectrum is determined by the interference of some trajectories with common photon energy [35]. Therefore, from Fig. 2 we find that the regular modulation in the upper branch $\mathrm{I}$ is due to the interference between the harmonics from short and long trajectories of only the pair $\mathrm{C}$ with electron recollision energy 


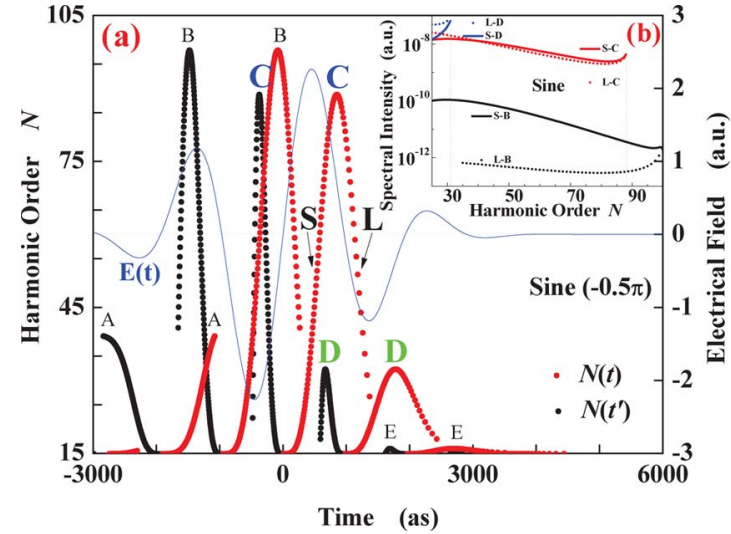

Fig. 2. (Color online) (a) Electron kinetic energy as functions of emission (thick black dotted curves) and recombination (thick red dotted curves) times with the electrical field (thin blue curve) by monocycle-sine pumping; (b) spectral intensity of each short (dashed curves) and long (solid curves) trajectories of three main pairs of quantum orbits (B, C and D) by monocycle-sine pumping.

from a fixed $E_{K}^{C}$ [corresponding to the boundary $N_{b}^{I}$ of the branch I, which is $N_{\max }^{D}=32$ in Fig. 2(a)] to the maximum $E_{K, \max }^{C}$ [corresponding to the peak $\overline{N_{\text {peak }}^{I}}$ of the branch I, which is $N_{\max }^{C}=88$ in Fig. 2(a)]. This is because the spectral intensity from the short trajectory of pair B [black solid curve S-B in Fig. 2(b)] and that from the long trajectory (black dotted curve L-B) are very low (less than $1 \%$ ), due to the low electron tunneling rate from pair $\mathrm{B}$ as a result of the low temporal electric field at the ionization time $t^{\prime}[28,37,38]$.

Here let us consider the origin of the steplike spectral phase behavior. We also take up the upper branch I at CEP $=-0.5 \pi$ as an example [thin red solid curve $\phi_{S . L}(\omega)$ in Fig. 3]. Using the spectral phase $\phi_{S-C}(\omega)$ (thick red solid curve in Fig. 3) and the intensity spectrum $I_{S-C}(\omega)$ (thick black solid curve in Fig. 3) from the short trajectory of pair $\mathrm{C}$ as well as the correspond-

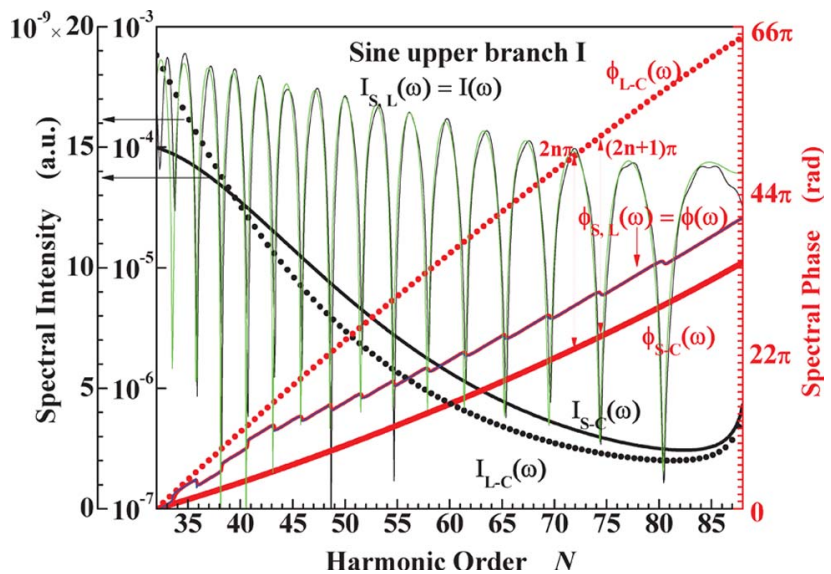

Fig. 3. (Color online) Spectral intensity and phase of short $\left[I_{S-C}(\omega)\right.$ and $\left.\phi_{S-C}(\omega)\right]$ and long $\left[I_{L-C}(\omega)\right.$ and $\left.\phi_{L-C}(\omega)\right]$ trajectories of the quantum orbit $C$ from SPA by monocycle-sine pumping. The spectral phase $\phi_{S-C}(\omega)$ (thick red solid curve) and the intensity spectrum $I_{S-C}(\omega)$ (thick black solid curve) from the short trajectory of the quantum orbit $\mathrm{C}$ as well as the corresponding spectral phase $\phi_{L-C}(\omega)$ (thick red dotted curve) and intensity spectrum $I_{L_{C}}(\omega)$ (thick black dotted curve) from the long trajectory; the calculated spectral phase $\phi_{S L}(\omega)$ [thin blue solid curve; from Eq. (3)] and intensity spectrum $I_{S, L}(\omega) \propto\left|E_{S, L}(\omega)\right|^{2}$ [thin green solid curve from Eq. (2)] show an excellent agreement with the steplike spectral phase of the high-order harmonics $\phi(\omega)$ (thin red solid curve) and the modulated intensity spectrum $I(\omega)$ (thin black solid curve). ing spectral phase $\phi_{L-C}(\omega)$ (thick red dotted curve in Fig. 3) and intensity spectrum $I_{L-C}(\omega)$ (thick black dotted curve in Fig. 3) from the long trajectory, we can obtain the combined complex electric-field spectrum:

$$
\begin{aligned}
E_{S, L}(\omega)= & I_{S-C}(\omega)^{1 / 2} \exp \left(i \phi_{S-C}(\omega)\right) \\
& +I_{L-C}(\omega)^{1 / 2} \exp \left(i \phi_{L-C}(\omega)\right) \\
= & {\left[I_{S-C}(\omega)+I_{L-C}(\omega)+2\left(I_{S-C}(\omega) \times I_{L-C}(\omega)\right)^{1 / 2}\right.} \\
& \left.\times \cos \phi_{0}(\omega)\right]^{1 / 2} \times \exp \left(i \phi_{S, L}(\omega)\right),
\end{aligned}
$$

where the spectral phase is

$$
\begin{aligned}
& \phi_{S, L}(\omega) \\
& =\tan ^{-1}\left\{\frac{\left[I_{S-C}(\omega)^{1 / 2} \sin \phi_{S-C}(\omega)+I_{L-C}(\omega)^{1 / 2} \sin \phi_{L-C}(\omega)\right]}{\left[I_{S-C}(\omega)^{1 / 2} \cos \phi_{S-C}(\omega)+I_{L-C}(\omega)^{1 / 2} \cos \phi_{L-C}(\omega)\right]}\right\},
\end{aligned}
$$

and $\phi_{0}(\omega)=\phi_{S-C}(\omega)-\phi_{L-C}(\omega)$. The calculated spectral phase $\phi_{S, L}(\omega)$ (thin blue solid curve in Fig. 3) and intensity spectrum $I_{S L}(\omega) \propto\left|E_{S L}(\omega)\right|^{2}$ (thin green solid curve in Fig. 3) showed an excellent agreement with the steplike spectral phase of the high-order harmonics $\phi(\omega)$ (thin red solid curve in Fig. 3) and the modulated intensity spectrum $I(\omega)$ (thin black solid curve in Fig. 3), which were obtained from the Lewenstein model, respectively. The phase step and the local minimum of the intensity spectrum occur at the deconstructive interference condition $\left[(2 n+1) \pi=\phi_{S-C}\left(\omega_{\min }^{(n)}\right)-\phi_{L-C}\left(\omega_{\min }^{(n)}\right)\right]$, while the local maximum of the intensity spectrum occurs at the constructive interference $\left[2 n \pi=\phi_{S-C}\left(\omega_{\max }^{(n)}\right)-\phi_{L-C}\left(\omega_{\max }^{(n)}\right)\right]$. Application of the distribution data of the phase frequency values satisfying this relation between the $\left(2 n \pi=\phi_{0}\left(\omega_{\max }^{(n)}\right)\right.$, $\left.(2 n+1) \pi=\phi_{0}\left(\omega_{\min }^{(n)}\right)\right)$ and the $\left(\omega_{\max }^{(n)}, \omega_{\min }^{(n)}\right)$ for different $n$ to the measured intensity spectrum $I(\omega)$ in the upper branch may enable us to obtain experimental information on the spectral phase at different CEPs and the CEP of the pump electric field.

Finally, we show the temporal profile $I(t)$ of the pulse (Fig. 4) generated from the harmonic wave with the total bandwidth (from $N_{b}^{I}=32$ to $N_{\text {peak }}^{I}=88$ ) of the upper branch I at $\mathrm{CEP}=-0.5 \pi$ [see the intensity spectrum $I(\omega)$ of the thin

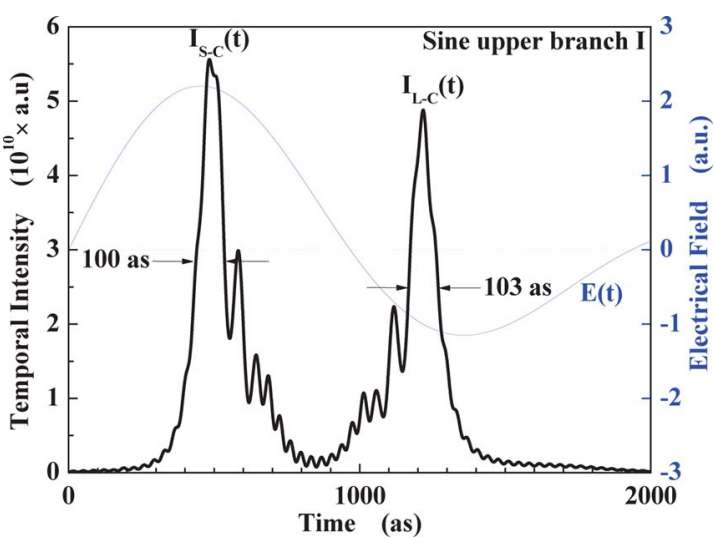

Fig. 4. (Color online) Temporal intensity profile [thick black curve, $I_{S-C}(t)$ and $I_{L-C}(t)$ components] obtained by the IFT at the same spectral bandwidth (from upper-branch thirty-second to eighty-eighth orders) with the electrical field curve $E(t)$ (blue curve) by monocyclesine pumping. 
black solid curve and the spectral phase $\phi(\omega)$ of the thin red solid curve in Fig. 3]. A double-peak pulse is produced, where the duration of the first subpulse is 100 as (without satellite peak), and the intensity is higher than that of the second subpulse [20]. The corresponding transform-limited (TL) pulse duration $\left(t_{\mathrm{TL}}\right)$ is 31 as. At all CEPs, the double-peak pulse was observed within a half-cycle time of the pump field. From comparison between Figs. 2(a) and $\underline{4}$ with the pump field profile $E(t)$, we find that the first $\left[I_{S-C}(t)\right]$ and the second $\left[I_{L-C}(t)\right]$ subpulses are assigned to be originated from the short and long trajectories of pair $\mathrm{C}$, respectively. The reason why the intensity of the $I_{S-C}(t)$ is higher than that of the $I_{L-C}(t)$ is that the spectral intensity $I_{S-C}(\omega)$ from the S-C trajectory is higher than that of the $I_{L-C}(\omega)$ from the $\mathrm{L}-\mathrm{C}$ trajectory, as shown in Figs. 2(b) or 3. The feature presented in Figs. $\underline{1}-\underline{4}$ (spectral intensity, phase, and temporal profile) was also confirmed by the TDSE simulation where the effect of the groundstate depletion was exactly considered $\left[|a(t)|^{2} \neq 1\right]$. While we have focused on the case of $\phi_{0}=-0.5 \pi$, we have also found essentially similar behaviors for different CEPs.

\section{ISOLATED ATTOSECOND PULSE SHORTENING: THE INTRINSIC DISPERSION EFFECT}

It has been well established that the short trajectory of all pairs of orbits is separated from the long trajectory using the following technique in experiment $[\underline{3}, \underline{4}, \underline{6}, \underline{8}, \underline{17}, 21-27]$ : the gas target is located at the defocusing position behind the focusing point of the visible pump beam, and then the outer part of the harmonic beam cross section is blocked by an aperture. In such harmonic generation conditions, the macroscopic response contains only the short trajectory contributions, which results in regular harmonic spectra and phases predicted by the single-atom response because the harmonic emission strength from the long trajectories is less than the short trajectories due to electron wave packet spreading and is much more sensitive to the pump pulse intensity [20-27]. This enables us to investigate the IAP generation using harmonic spectra and intrinsic dispersions from only the short trajectory of the quantum orbit at single-atom level [22]. Figure 5 shows the harmonic intensity spectra of the upper branch $\overline{\mathrm{I}}$

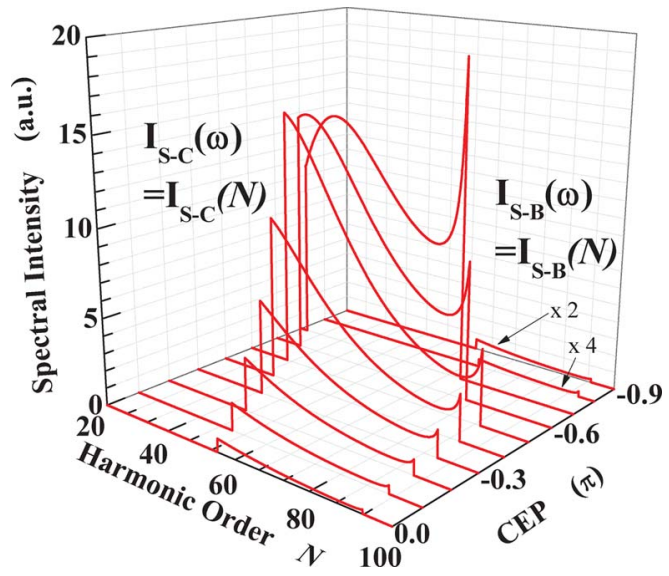

Fig. 5. (Color online) The intensity spectra $I_{S-C}(\omega)$ in the upper branch I from CEP $=0.0 \pi$ to $-0.7 \pi$ and those $I_{S-B}(\omega)$ in the upper branch II from CEP $=-0.8 \pi$ to $-0.9 \pi$ (for easy comparison, we increased the intensity to four and two times at CEP $=-0.8 \pi$ and $-0.9 \pi$, respectively), which were calculated from the SPA. or II from only the single short trajectory S-C or S-B for different CEPs, which are taken out by suitable filters [17,39]. The filters can be implemented by a suitable combination of one-stacking or double-stacking [40] two-period multilayer mirrors consisting of pair layers of two materials (heavy/light elements such as $\mathrm{Mo} / \mathrm{B}_{4} \mathrm{C}$ and $\left.\mathrm{Mo} / \mathrm{Si}[\underline{17}, \underline{39}, \underline{41}]\right)$ with a ratio between the material thicknesses, a one-period thickness and a layer number, which are adjusted for the desired center wavelength, bandwidth and reflectivity at an optimum incident angle, and of thin metal foils such as $\operatorname{Zr~[17].~The~intensity~}$ spectra $I_{S-C}(\omega)$ in the upper branch I from CEP $=0.0 \pi$ to $-0.7 \pi$ and those $I_{S-B}(\omega)$ in the upper branch II from CEP $=$ $-0.8 \pi$ to $-0.9 \pi$ were calculated using the SPA [see Figs. $2(\mathrm{~b})$ and 3 for CEP $=-0.5 \pi$ ]. Those spectra can be measured experimentally by an XUV spectrometer. The reason why the spectra of the upper branch II for CEP $=-0.8 \pi$ and $-0.9 \pi$ were selected is as follows: for those CEPs, two spectra in the upper branch I from two short trajectories of the pairs $\mathrm{C}$ and $\mathrm{B}$ appear (the latter spectral intensity is about $80 \%$ weaker) and hence always lead to a double pulse, which should be avoided for the IAP generation. The CEP dependence of the intensity spectrum $\left[I_{S-C}(\omega)\right.$ or $\left.I_{S-B}(\omega)\right]$ with an asymmetrical structure corresponds to the upper branch in Fig. 1 (its vertical axis is a logarithm scale) from the interference between the short and long trajectories. Since the spectral intensity rapidly increases with the present temporal electric field $E\left(t^{\prime}\right)$ at the ionization time $t^{\prime}$, it becomes largest at around $\mathrm{CEP}=-0.7 \pi$.

To get the shortest IAP without chirp compensation for the above-observed spectra by optimization of the spectral region and the CEP, we use the recombination time method (see Section 2) because this method provides a possibility for the experimental measurement of the intrinsic harmonic dispersion [23,27]: the recombination time as a function of the harmonic order $t(N)$ for the single electron trajectory [see Fig. 2(a) for $N(t)$ of the S-C trajectory at CEP $=-0.5 \pi]$ provides the quantitative information on the so-called intrinsic harmonic atto-chirp [23,27]. Since the function $t(N)$ [see Fig. 6(a)] corresponds to the group delay $t_{\mathrm{DG}}(\omega)$, defined as the first derivative of the spectral phase $\phi(\omega)$ with respect to the angular frequency $\omega$, the slope $d t(N) / d N$ is the GDD [ $\phi^{\prime \prime}(N)=d^{2} \phi(\omega) / d \omega^{2}$; see Fig. 6(b)] [31,33,34], which broadens the attosecond pulse duration. By carrying out the inverse Fourier transform (IFT) using the harmonic-order-dependent GDD $\phi_{S-j}^{\prime \prime}(N)$ [see Fig. $\underline{6(\mathrm{~b})}$ ] and the intensity spectrum $I_{S-j}(N)$ from the single short trajectory ( $j=\mathrm{C}$ or B; Fig. 5 ), we can obtain the temporal profile and duration $t_{p}$ of the IAP for different spectral ranges and CEPs. The recombination time method has an advantage in that we can easily and directly deduce the optimum spectral range from the GDD curve $\phi^{\prime \prime}(N)$ as a harmonic region having the minimum GDD and higher-order dispersion before the practical IFT calculation.

Figures $\underline{6(\mathrm{a})}$ and $\underline{6(\mathrm{~b})}$ show the calculated recombination time $t(N)$ from the $\overline{\mathrm{S}-\mathrm{C}}$ or $\mathrm{S}-\mathrm{B}$ trajectory from $\mathrm{CEP}=0.0 \pi$ to $-0.9 \pi$ and the corresponding positive GDD $\phi_{S-j}^{\prime \prime}(N)$, respectively. Figure $6(\mathrm{~b})$ suggests that the GDD increases rapidly with closing to the cutoff region, while Fig. 5 suggests that the harmonic intensity decreases rapidly with closing to the cutoff region except for the $\mathrm{CEP}=-0.6 \pi$ and $-0.7 \pi$. From Fig. 6(b), we can select the favorable spectral range and CEP, which satisfy the condition that the GDD value at the 


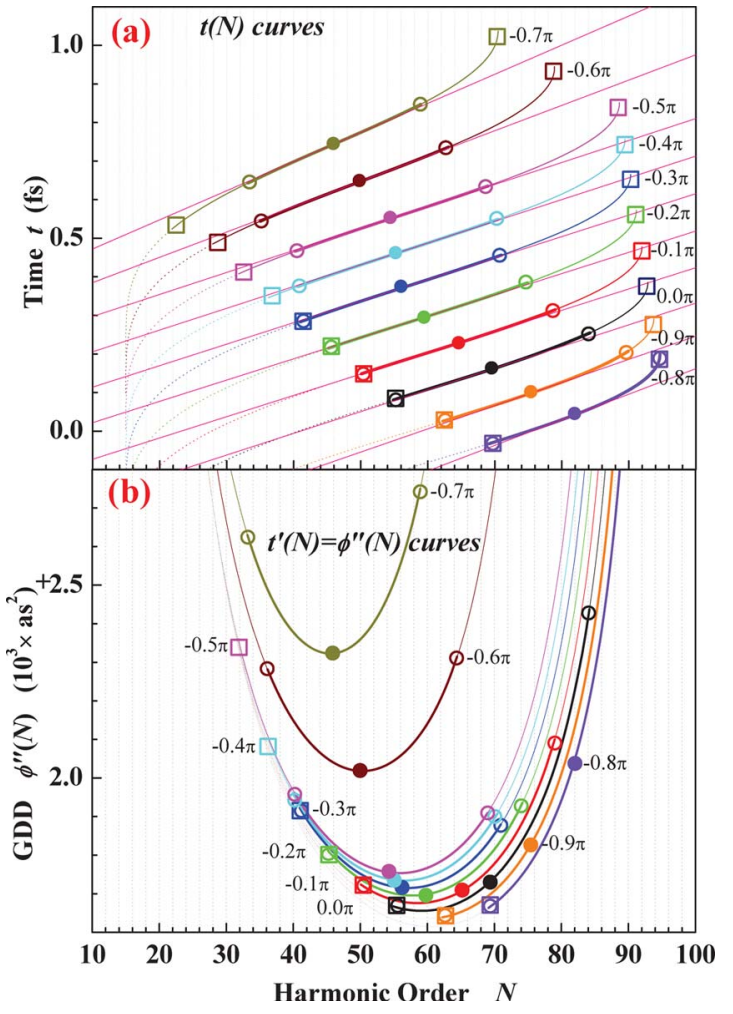

Fig. 6. (Color online) (a) Harmonic-order-dependent recombination time $t(N)$ from the S-C or S-B trajectory from CEP $=0.0 \pi$ to $-0.9 \pi$, which was calculated from the simple semiclassical method; (b) the corresponding $N$-dependent positive GDD $\phi_{S-i}^{\prime \prime}(N)$. For both (a) and (b), dotted lines, thin solid lines, and thick solid lines show the total trajectories, the upper branch and the optimum region, respectively, and the open square $(\square)$, open circle $(\bigcirc)$, and solid circle $(O)$ show the ends of upper branch, the ends of optimum region, and the center of optimum region, respectively.

center harmonic order $N_{C}$ is as small as possible, and the $N$ dependent GDD is as constant as possible within the region of the upper branch [from $22-70$ to 69-96 marked by open squares $(\square)$ in Figs. 6(a) and $\underline{6(b)}$ ]. Figures $\underline{7(a)}$ and $\underline{7(b)}$ show the typical dependence of the pulse profile and duration (inset) on the bandwidth $\Delta N$ at a constant $N_{C}=54.5$ and that on the $N_{C}$ at a constant $\Delta N=29$ for CEP $=-0.5 \pi$, respectively [see CEP $=-0.5 \pi$ in Figs. $\underline{5}$ and $\underline{6(b)}$ ]. In the former, the duration rapidly decreases from 170 to 68 as, with the increase of $\Delta N$ from 10 to 29 , and with the further increase from $\Delta N=32$ to 46 , the pulse splits and broadens mainly due to the large third-order dispersion (TOD) and higher-order dispersion. While in the latter, the duration slightly changes around 68 to 69 as with the increase of $N_{C}$ from 46.5 to 60 , and with the further increase from $N_{C}=65$ to 70.5 , it somewhat increases to 80 as due to the combined effect of the decrease of the spectral intensity and the increase of the positive GDD and TOD. The obtained shortest pulse profiles at each CEP and the corresponding CEP dependence of the pulse duration are shown in Figs. 8(a) and 8(b), respectively, where

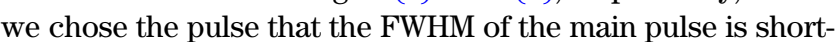
est among all the calculated pulses. The shortest pulse duration $t_{p}(\mathrm{FWHM})$ at each CEP and the corresponding spectral range are summarized in Table 1 . Those spectral regions are shown by bold solid curves from 33-59 to 69-95, marked by open circles (O), in Figs. 6(a) and 6(b). As a result, we find that the frequency region having the minimum GDD and
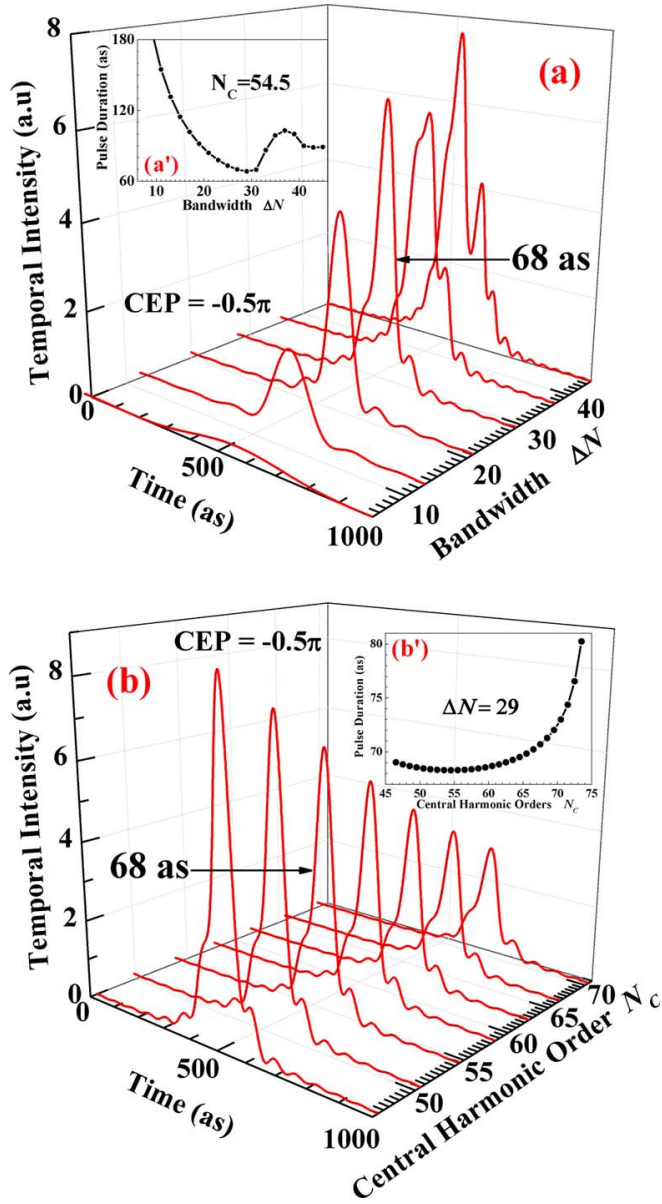

Fig. 7. (Color online) (a) Pulse profile and duration [inset Fig. 7(a)'] on as a function of the bandwidth $\Delta N$ at a constant central harmonic order $N_{C}=54.5$ for CEP $=-0.5 \pi$; (b) pulse profile and duration [inset Fig. 7(b)'] on as a function of the central harmonic order $N_{C}$ at a constant bandwidth $\Delta N=29$ for CEP $=-0.5 \pi$.

higher-order dispersion within the upper branch for all the CEPs was chosen as an optimum spectral range [see Fig. 6(b)]. From Fig. 8(a), we find that the shortest IAP with a 68 as duration $\left(t_{p}\right)$ can be generated without chirp compensation under the spectral range of $45-74$ at CEP $=-0.2 \pi$ and under the spectral range of $41-71$ at $\mathrm{CEP}=-0.3 \pi$ [ $\phi^{\prime \prime}\left(N_{c}\right)=+1.7 \times 10^{3}$ as $^{2}: t_{\mathrm{TL}}=58$ as]. Furthermore, Fig. $\underline{8(\mathrm{~b})}$ suggests that tunable (from 115 to $150 \mathrm{eV}$ ), sub 70 as isolated pulses can be generated by tuning the CEP from $0.0 \pi$ to $-0.5 \pi$, with the increase of the XUV pulse intensity (from $0.32 \mathrm{a} . \mathrm{u}$. to 6.55 a.u.). It should be noted that the shortest IAP is generated in the middle frequency region of the plateau at CEP $=-0.2 \pi$ and $-0.3 \pi$, corresponding to the minimum GDD and higherorder dispersion region. This is completely different from the well-known fact for few-cycle pumping that the shortest IAP has been generated in the cutoff region at CEP $=0.0 \pi$ (cosine) $[\underline{1}, \underline{3}, \underline{4}, \underline{21}]$, where the harmonic intensity drops rapidly. In addition, our optimum frequency region is also different from the recent experimental result of sub 1.5 cycle pumping [17], where the frequency region selected with chirp compensation is from the cutoff region to its close plateau region, but the selected CEP is almost the same. The green solid curve in Fig. 9(a) shows the shortest pulse profile $I_{S-C}(t)$ $\left(t_{p}=\overline{73 \mathrm{as}}, t_{\mathrm{TL}}=62\right.$ as) obtained at $N_{H}-N_{L}=90-63$ and $\mathrm{CEP}=-0.3 \pi$, where the spectrum contains the cutoff region 


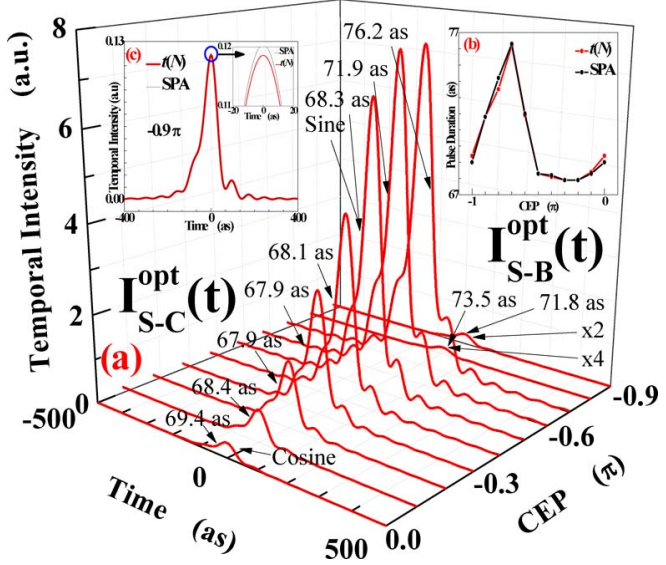

Fig. 8. (Color online) (a) Temporal profile of the shortest IAP for different CEPs (thin black solid curves are from SPA and thick red solid curves are from $t(N)$; they are exactly overlapped, so are not easily distinguished and then only the red solid curves are shown); (b) CEP dependence of the pulse duration (black curve is from SPA and red curve is from $t(N)$ ); (c) enlarged temporal profile of the shortest IAP for CEP $=-0.9 \pi$ [here we can see the difference between the SPA and $t(N)$ from the inset figure].

[17], and the blue solid curve in Fig. 9(a) shows the $I_{S-C}(t)$ $\left(\overline{t_{p}}=82\right.$ as, $t_{\mathrm{TL}}=41$ as) obtained at $\overline{N_{H}}-N_{L}=92-50$ and CEP $=-0.1 \pi$, where the spectrum contains the entire upper branch $\left(N_{H}\right.$ and $N_{L}$ are the highest and lowest harmonic order edges). Correspondingly, the green solid curve in Fig. 9(b) shows the GDD curve $\phi^{\prime \prime}(N)$ in the former case [GDD at $N_{C}, \phi^{\prime \prime}\left(N_{c}\right)=+2.0 \times 10^{3} \mathrm{as}^{2}$ : TOD at $N_{C}, \phi^{\prime \prime \prime}\left(N_{c}\right)=+1.57 \times$ $10^{4} \mathrm{as}^{3}$ ], and the blue solid curve in Fig. 9(b) shows the $\phi^{\prime \prime}(N)$ in the latter case $\left[\phi^{\prime \prime}\left(N_{c}\right)=+1.8 \times 10^{3}\right.$ as $^{2}: \phi^{\prime \prime \prime}\left(N_{c}\right)=$ $+0.6 \times 10^{4} \mathrm{as}^{3}$ ]. For comparison, we also show the shortest result (the red solid curves, $t_{p}=68$ as at $N_{H}-N_{L}=71-41$ and CEP $=-0.3 \pi)$, which is shown in Figs. 8(a) and 6(b). In both cases, the pulse broadens and splits due to the combined effect of the very large positive TOD at $N_{C}$ (more than 10 times compared with the shortest 68 as case) and the relatively weak intensity of the related spectral part.

Here let us examine the reliability of this recombination time method by comparing with the results of the SPA. That is, we also obtained the temporal profile of the shortest IAP for different CEPs [thin black solid curves in Fig. 8(a) and enlarged one in Fig. $\underline{8(\mathrm{c})}$ for CEP $=-0.9 \pi]$ and the corresponding CEP dependence of the pulse duration [thin black solid curve in Fig. 8(b)]. In addition, in Table 1, we show the values of the parameters obtained from the latter methods, such as $t_{p}, \phi_{S-j}^{\prime \prime}\left(N_{c}\right)$, and $\phi_{S-j}^{\prime \prime \prime}\left(N_{c}\right)$. All the results show the excellent

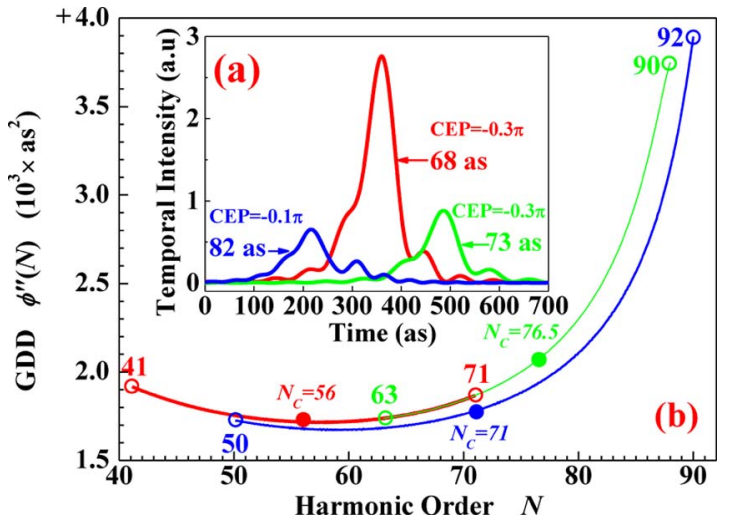

Fig. 9. (Color online) (a) Shortest pulse profile after the optimum selection in three different cases; (b) the corresponding GDD $\phi^{\prime \prime}(N)$ curve. Those were obtained by $N_{H}-N_{L}=90-63\left(N_{H}\right.$ and $N_{L}$ are the highest and lowest harmonic-order edges, respectively) and CEP $=-0.3 \pi$, where the spectrum contains the cutoff region (thin green curves); by $N_{H}-N_{L}=92-50$ and CEP $=-0.1 \pi$, where the spectrum contains the entire upper branch (thick blue curves); and by $N_{H}-N_{L}=71-41$ and CEP $=-0.3 \pi$, where the spectrum contains the plateau region only (thick red curves), respectively. The open circle $(\mathrm{O})$ and solid circle $(-)$ show the ends of optimum region and the center of optimum region, respectively.

agreement for all CEPs, definitely supporting the validity of the recombination time method.

We also found using the recombination time method that monocycle pumping can generate efficiently the isolated pulse with a six times higher intensity (a 68 as duration at the $\mathrm{CEP}=0.5 \pi$ ) than that (a 150 as duration at the optimum $\mathrm{CEP}=0.0 \pi$ ) of two-cycle (TC) pumping with the double energy. The latter pulse duration is close to the experimental result of TC pumping $[\underline{1}, \underline{4}, 21]$.

Finally, we briefly describe examples of the application of the recombination time method to case $\mathrm{A}$ of the doubleintensity pump $\left(I_{\text {pump }}=2 I_{0}\right)$ and case B of the doublewavelength $\left(\lambda_{\text {pump }}=2 \lambda_{0}\right)$ and double-intensity $\left(I_{\text {pump }}=2 I_{0}\right)$ pump ( $T_{\text {pump }}=2 T_{0}$ ) because we can expect the generation of the shorter IAP from the relation between the pump intensity (or the pump wavelength) and the recombination time $t(N)$. The red solid curve in Fig. 10(a) and that in Fig. 10(b) show the pulse profile $I_{S-C}^{\mathrm{opt}}(t)$ and the GDD $\phi_{S-C}^{\prime \prime}(N)$ at $\overline{\mathrm{EP}}=$ $-0.3 \pi$ after the optimum selection of the spectral range, which is the middle spectral region of the plateau, $N_{H}-N_{L}=119-81$ (thick red part) for case A, respectively. Correspondingly, the blue solid curve in Fig. 10(a) and that in Fig. 10(b) show the $I_{S-C}^{\text {opt }}(t)$ and the $\phi_{S-C}^{\prime \prime}(N)$ at CEP $=-0.3 \pi$ after the optimum selection of the spectral range, $N_{H \text {.eff }}-N_{L \text {.eff }}=370-320.5$ (thick blue part, $N_{H . \text {.eff }}=N_{H} / 2$ and $N_{L \text {.eff }}=N_{L} / 2$ ) for

Table 1. Shortest Pulse Duration $t_{p}$ and the Corresponding GDD, TOD, and Spectral Range $\left(N_{H}-N_{L}\right)$ Obtained by Two Methods $[t(N)$ and SPA] for Each CEP

\begin{tabular}{|c|c|c|c|c|c|c|c|c|c|c|c|}
\hline $\operatorname{CEP}(\pi)$ & & 0.0 & -0.1 & -0.2 & -0.3 & -0.4 & -0.5 & -0.6 & -0.7 & -0.8 & -0.9 \\
\hline$t_{p}$ (as) & SPA & 69.0 & 68.3 & 67.9 & 67.9 & 68.2 & 68.3 & 72.0 & 76.3 & 74.2 & 71.0 \\
\hline GDD at $N_{C}\left(10^{3} \times \mathrm{as}^{2}\right)$ & SPA & 1.72 & 1.69 & 1.70 & 1.72 & 1.74 & 1.76 & 2.02 & 2.33 & 1.97 & 1.81 \\
\hline \multirow[t]{2}{*}{ TOD at $N_{C}\left(10^{2} \times \mathrm{as}^{3}\right)$} & $t(N)$ & 50.64 & 27.15 & 7.61 & -4.96 & -6.96 & -6.17 & -2.50 & 5.19 & 186.2 & 93.24 \\
\hline & SPA & 45.24 & 24.03 & 6.28 & -6.31 & -6.34 & -7.16 & -8.66 & 7.26 & 154.6 & 84.68 \\
\hline
\end{tabular}




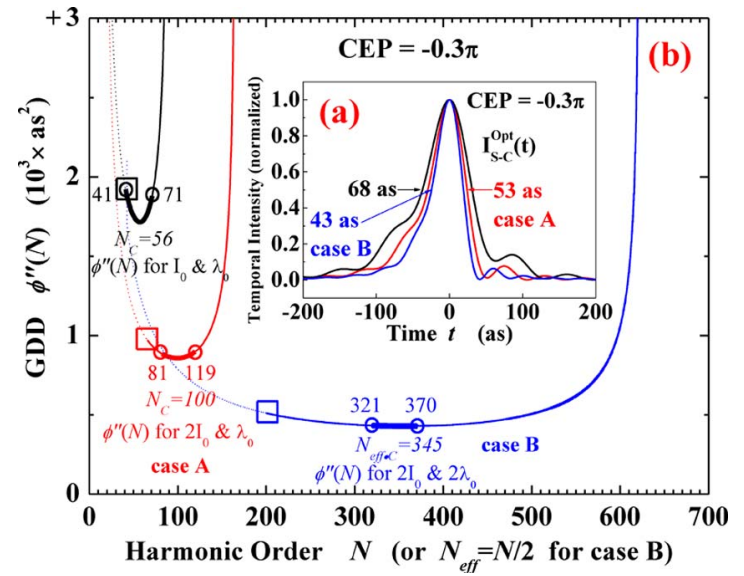

Fig. 10. (Color online) (a) Normalized pulse profile $I_{S-C}^{\text {opt }}(t)$ after the optimum selection; (b) $N$-dependent GDD $\phi_{S-C}^{\prime \prime}(N)$ curves at CEP $=-0.3 \pi$ : black curve for the intensity $\left(I_{0}\right)$ and wavelength $\left(\lambda_{0}\right)$ pump case, red curve for the double-intensity $\left(2 I_{0}\right)$ and wavelength $\left(\lambda_{0}\right)$ pump case, and blue curve for the double-intensity $\left(2 I_{0}\right)$, double-wavelength $\left(2 \lambda_{0}\right)$ pump case; $\left(N_{\text {eff }}=N / 2\right)$. The open square $(\square)$ and open circle $(O)$ show the ends of upper branch and the ends of optimum region, respectively.

case $\mathrm{B}$, respectively. For comparison, the corresponding $I_{S-C}^{\text {opt }}(t)$ and $\phi_{S-C}^{\prime \prime}(N)$ at the pump intensity $I_{\text {pump }}=I_{0}$, the pump wavelength $\lambda_{\text {pump }}=\lambda_{0}$, and CEP $=-0.3 \pi$ [the black solid curves; see Figs. 8(a) and 6(b) ] are also shown. We find that the shorter 53 as isolated pulse for case $A$ and the 43 as pulse for case B, which are close to the TL pulse (47 as and 39 as), can be generated with the smaller positive GDD $\left(+8.6 \times 10^{2} \mathrm{as}^{2}\right.$ and $+4.3 \times 10^{2}$ as $^{2}$ at $N_{C}$ ) without chirp compensation. This result was also confirmed by the SPA. Moreover, we confirmed that the result is reliable from the corresponding TDSE calculation, exactly considering the contributions from the short trajectory and the long trajectory: only the slightly shorter attosecond, high-intensity pulse $I_{S-C}(t)$ from the short trajectory and the much lower intensity pulse $I_{L-C}(t)$ from the long trajectory were observed at the reasonable time interval for both cases. The GDD curve $\phi^{\prime \prime}(N)$ as a function of the harmonic order $N$ indicates that the GDD decreases and the plateau frequency region broadens toward the higher frequency with the increase of the pump intensity and the pump wavelength [23,27] (under the condition that the pump field cycle does not change so as not to change the intrinsic profile of its curve), suggesting the generation of the shorter IAP close to the Fourier transform limitation. Obviously, the long-wavelength pumping results support the implicit predictions for the shorter IAP generation. However, it also accompanies the dramatic decrease in efficiency [12]. From an application viewpoint, we maybe face the trade-off between the shorter pulse duration and the accessible harmonic yield, but this problem will be solved by some way.

\section{CONCLUSION}

We have clarified that the harmonic wave generated from He by monocycle pumping $\left(T_{0}=1.93 \mathrm{fs}, \quad \lambda_{0}=580 \mathrm{~nm}\right.$, $I_{0}=1.70 \sim 2.00 \times 10^{15} \mathrm{~W} / \mathrm{cm}^{2}, \quad$ CEP $=0.0 \pi$ to $-0.9 \pi$ and Gaussian shape) has distinguished features: the high-order harmonic spectrum consisting of one or two upper branches with several regular and slow modulations, the spectral phase with the regular steplike changes in the upper branch, and the pulse profile having a double-peak shape within the half-cycle time. These characteristics have been explained by the interference between the harmonics emitted from the short trajectory and that from the long trajectory of only the single pair quantum orbit.

Furthermore, we have shown that it is essential for the generation of the shorter isolated pulse without chirp compensation, not only to utilize the upper-branch harmonics emitted from only the single short trajectory by the technique of the trajectory selection, but also to select the harmonics of the spectral range having the minimum GDD and higher-order dispersion in the middle spectral region of the plateau where the harmonic intensity is high. For the latter selection, we have demonstrated that the calculation result of the first derivative of the recombination time $t^{\prime}(N)$, which corresponds to the general frequency-dependent GDD in optics, is very useful in monocycle pumping for the prediction of the optimum spectral region as well as the exact values of the GDD and higherorder dispersion. In addition, $t(N)$ is calculated by the simple semiclassical three-step model and has a possibility to be measured experimentally. The result of the IFT using the GDD $t^{\prime}(N)=\phi^{\prime \prime}(N)$ and the harmonic spectrum (which can also be measured) from the short trajectory for different CEPs suggests that we can generate a 68 as isolated pulse with the spectrum in the plateau's middle region (not close to the cutoff region) of $N=45$ to 74 at $\mathrm{CEP}=-0.2 \pi$ and that of $N=41$ to 71 at $\mathrm{CEP}=-0.3 \pi$ without chirp compensation. This result has also been confirmed by the SPA approach. Moreover, the result suggests that we can realize an XUV source of 115 to $150 \mathrm{eV}$ tunable, sub 70 as isolated pulses by tuning the CEP. Also, it has been shown that monocycle pumping can efficiently generate a 68 as isolated pulse with six time higher intensity than that of the 150 as pulse of TC pumping. Finally, the application of the recombination time method to monocycle pumping with double intensity $\left(2 I_{0}\right)$ and with double wavelength $\left(2 \lambda_{0}\right)$ and intensity $\left(2 I_{0}\right)$ has shown that we can generate the 53 as isolated pulse and the 43 as pulse close to the TL pulse (47 as and 39 as) without chirp compensation, respectively.

\section{ACKNOWLEDGMENTS}

This work was mainly supported by the Core Research for Evolutional Science and Technology, Japan Science and Technology Agency (JST-CREST). K. L. I. gratefully acknowledges financial support from the Advanced Photon Science Alliance (APSA) project (Japan). S. F. gratefully acknowledges financial support from the China Scholarship Council (CSC).

\section{REFERENCES}

1. F. Krausz and M. Ivanov, "Attosecond physics," Rev. Mod. Phys. 81, 163-234 (2009).

2. P. Agostini and L. F. DiMauro, "The physics of attosecond light pulses," Rep. Prog. Phys. 67, 813-855 (2004).

3. M. Hentschel, R. Kienberger, C. Spielman, G. A. Reider, N. Milosevic, T. Brabec, P. Corkum, U. Heizmann, M. Drescher, and F. Krausz, "Attosecond metrology," Nature 414, 509-513 (2001).

4. R. Kienberger, E. Goulielmakis, M. Uiberacker, A. Baltuska, V. Yakovlev, F. Bammer, A. Scrinzi, T. Westerwalbesloh, U. Kleineberg, U. Heinzmann, M. Drescher, and F. Krausz, "Atomic transient recorder," Nature 427, 817-821 (2004).

5. O. Tcherbakoff, E. Mével, D. Descamps, J. Plumridge, and E. Constant, "Time-gated high-order harmonic generation," Phys. Rev. A 68, 043804 (2003). 
6. G. Sansone, E. Benedetti, F. Calegari, C. Vozzi, L. Avaldi, R. Flammini, L. Poletto, P. Villoresi, C. Altucci, R. Velotta, S. Stagira, S. De Silvestri, and M. Nisoli, "Isolated single-cycle attosecond pulses," Science 314, 443-446 (2006).

7. T. Pfeifer, L. Gallmann, M. J. Abel, D. M. Neumark, and S. R. Leone, "Single attosecond pulse generation in the multicycledriver regime by adding a weak second-harmonic field," Opt. Lett. 31, 975-977 (2006).

8. Z. N. Zeng, Y. Cheng, X. H. Song, R. X. Li, and Z. Z. Xu, "Generation of an extreme ultraviolet supercontinuum in a two-color laser field," Phys. Rev. Lett. 98, 203901 (2007).

9. T. Sekikawa, A. Kosuge, T. Kanai, and S. Watanabe, "Nonlinear optics in the extreme ultraviolet," Nature 432, 605-608 (2004).

10. A. Jullien, T. Pfeifer, M. J. Abel, P. M. Nagel, M. J. Bell, D. M. Neumark, and S. R. Leone, "Ionization phase-match gating for wavelength-tunable isolated attosecond pulse generation," Appl. Phys. B 93, 433-442 (2008).

11. V. S. Yakovlev, M. Ivanov, and F. Krausz, "Enhanced phasematching for generation of soft x-ray harmonics and attosecond pulses in atomic gases," Opt. Express 15, 15351-15364 (2007).

12. P. Colosimo, G. Doumy, C. I. Blaga, J. Wheeler, C. Hauri, F. Catoire, J. Tate, R. Chirla, A. M. March, G. G. Paulus, H. G. Muller, P. Agostini, and L. F. DiMauro, "Scaling strong-field interactions towards the classical limit," Nat. Phys. 4, 386-389 (2008).

13. L. E. Chipperfield, J. S. Robinson, J. W. G. Tisch, and J. P. Marangos, "Ideal waveform to generate the maximum possible electron recollision energy for any given oscillation period," Phys. Rev. Lett. 102, 063003 (2009).

14. W. Hong, P. Lu, W. Cao, P. Lan, and X. Wang, "Control of quantum paths of high-order harmonics and attosecond pulse generation in the presence of a static electric field," J. Phys. B 40, 2321-2331 (2007).

15. I. A. Ivanov and A. S. Kheifets, "Tailoring the waveforms to extend the high-order harmonic generation cutoff," Phys. Rev. A 80, 023809 (2009).

16. H. Mashiko, S. Gilbertson, C. Li, S. D. Khan, M. M. Shakya, E. Moon, and Z. Chang, "Double optical gating of high-order harmonic generation with carrier-envelope phase stabilized lasers," Phys. Rev. Lett. 100, 103906 (2008).

17. E. Goulielmakis, M. Schultze, M. Hofstetter, V. S. Yakovlev, J. Gagnon, M. Uiberacker, A. L. Aquila, E. M. Gullikson, D. T. Attwood, R. Kienberger, F. Krausz, and U. Kleineberg, "Singlecycle nonlinear optics," Science 320, 1614-1617 (2008).

18. E. Matsubara, K. Yamane, T. Sekikawa, and M. Yamashita, "Generation of $2.6 \mathrm{fs}$ optical pulses using induced-phase modulation in a gas-filled hollow fiber," J. Opt. Soc. Am. B 24, 985-989 (2007).

19. K. Yamane, T. Tanigawa, T. Sekikawa, and M. Yamashita, "Angularly-dispersed optical parametric amplification of optical pulses with one-octave bandwidth toward monocycle regime," Opt. Express 16, 18345-18353 (2008).

20. L. E. Chipperfield, L. N. Gaier, P. L. Knight, J. P. Marangos, and J. W. G. Tisch, "Conditions for the reliable production of attosecond pulses using ultra-short laser-generated high harmonics," J. Mod. Opt. 52, 243-260 (2005).

21. A. Baltuska, T. Udem, M. Uiberacker, M. Hentschel, E. Goulielmakis, C. Gohle, R. Holzwarth, V. S. Yakovlev, A. Scrinzi, T. W. Hansch, and F. Krausz, "Attosecond control of electronic processes by intense light fields," Nature 421, 611-615 (2003).

22. Q. Li, P. Lu, W. Hong, Q. Zhang, and Z. Yang, "Propagation effects for attosecond ionization control of efficient broadband supercontinuum generation," Phys. Rev. A 80, 043417 (2009).

23. S. Kazamias and P. Balcou, "Intrinsic chirp of attosecond pulses: single-atom model versus experiment," Phys. Rev. A 69, 063416 (2004).
24. D. Lee, H. Shin, Y. Cha, K. Hong, J. Kim, and C. Nam, "Selection of high-order harmonics from a single quantum path for the generation of an attosecond pulse train," Phys. Rev. A 63, 021801 (2001).

25. P. Antoine, A. L'Huillier, and M. Lewenstein, "Attosecond pulse trains using high-order harmonics," Phys. Rev. Lett. 77, 12341237 (1996).

26. P. Salières, B. Carré, L. Le Déroff, F. Grasbon, G. G. Paulus, H. Walther, R. Kopold, W. Becker, D. B. Milosevic, A. Sanpera, and M. Lewenstein, "Feynman's path-integral approach for intense-laser-atom interactions," Science 292, 902-905 (2001).

27. G. Doumy, J. Wheeler, C. Roedig, R. Chirla, P. Agostini, and L. F. DiMauro, "Attosecond synchronization of high-order harmonics from midinfrared drivers," Phys. Rev. Lett. 102, 093002 (2009).

28. M. Lewenstein, P. Balcou, M. Y. Ivanov, A. L'Huillier, and P. B. Corkum, "Theory of high-harmonic generation by low-frequency laser fields," Phys. Rev. A 49, 2117-2132 (1994).

29. L. E. Chipperfield, P. L. Knight, J. W. G. Tisch, and J. P. Marangos, "Tracking individual electron trajectories in a high harmonic spectrum," Opt. Commun. 264, 494-501 (2006).

30. K. C. Kulander, K. J. Schafer, and J. L. Krause, "Time-dependent studies of multiphoton processes," in Atoms in Intense Laser Fields, M. Gavrila, ed. (Academic, 1992), pp. 247-300.

31. M. Yamashita, K. Yamane, and R. Morita, "Quasi-automatic phase-control technique for chirp compensation of pulses with over-one-octave bandwidth-generation of few- to mono-cycle optical pulses,” IEEE J. Sel. Top. Quantum Electron. 12, 213222 (2006).

32. P. B. Corkum, "Plasma perspective on strong field multiphoton ionization,” Phys. Rev. Lett. 71, 1994-1997 (1993).

33. M. Yamashita, R. Morita, and N. Karasawa, "Active chirp compensation for ultra broadband optical pulses," in Mono-Cycle Photonics and Optical Scanning Tunneling Microscopy, M. Yamashita, H. Shigekawa, and R. Morita, eds. (Springer, 2005), pp. 103-151.

34. J. C. Diels and W. Rudolph, "Femtosecond optics," in Ultrashort Laser Pulse Phenomena, 2nd ed., J. C. Diels and W. Rudolph, eds. (Academic, 2006), pp. 61-142.

35. G. Sansone, C. Vozzi, S. Stagira, and M. Nisoli, "Nonadiabatic quantum path analysis of high-order harmonic generation: role of the carrier-envelope phase on short and long paths," Phys. Rev. A 70, 013411 (2004).

36. E. Matsubara, T. Sekikawa, and M. Yamashita, "Generation of ultrashort optical pulses using multiple coherent anti-Stokes Raman scattering in a crystal at room temperature," Appl. Phys. Lett. 92, 071104 (2008).

37. G. L. Yudin and M. Y. Ivanov, "Nonadiabatic tunnel ionization: looking inside a laser cycle," Phys. Rev. A 64, 013409 (2001).

38. M. V. Ammosov, N. B. Delone, and V. P. Krainov, "Tunnel ionization of complex atoms and atomic ions in an alternating electromagnetic field," Sov. Phys. JETP 64, 1191-1194 (1986).

39. H. Kinoshita, K. Kurihara, and H. Takenaka, "Soft x-ray reduction lithography using multilayer mirrors,” Jpn. J. Appl. Phys. 30, 3048-3052 (1991).

40. M. Yamashita, M. Ishikawa, K. Torizuka, and T. Sato, "Femtosecond-pulse laser chirp compensated by cavity-mirror dispersion," Opt. Lett. 11, 504-506 (1986).

41. B. L. Henke, E. M. Gullikson, and J. C. Davis, "X-ray interactions: photoabsorption, scattering, transmission, and reflection at $E=50-30,000 \mathrm{eV}, Z=1-92$," At. Data Nucl. Data Tables 54, 181-342 (1993). 